\title{
Intraoperative radiation therapy in non-breast cancer patients: A report of 26 cases from Shiraz, south of Iran
}

\author{
Majid Akrami ${ }^{1}$, Hamid Nasrollahi*2 ${ }^{\mathbb{D}}$, Mostafa Vahabi ${ }^{1}$, Seyed Hassan Hamedi ${ }^{2}$, Sedigheh Tahmasebi ${ }^{1}$, Sareh $\mathrm{Karbasi}^{2}$, \\ Mehran Pashnesaz ${ }^{1}$, Vahid Zangouri ${ }^{1}$, Mohammad Yasin Karami ${ }^{1}$, Ahmad Mosallaei ${ }^{2}$, Abdolrasoul Talei ${ }^{1}$
}

Received: 6 Oct 2019

Published: 20 May 2020

\section{Abstract}

Background: Intraoperative radiation therapy (IORT) is the delivery of radiation at the time of surgery. Whereas the dose delivered by external beam radiation therapy (EBRT) is limited by the tolerance of the surrounding normal tissues, IORT allows exclusion of a part or all of the dose-limiting sensitive structures by operative mobilization and/or direct shielding of these structures. The aim of the present study was to report the non-breast cancer patients' outcomes after receiving IORT in Shiraz, Iran.

Methods: In this retrospective study, all cases who had received IORT and had non-breast malignancies were selected. Diagnosis was confirmed by biopsy. Additional imaging was done by sonography, magnetic resonance imaging (MRI) and computed tomography (CT). IORT was applied by self-shielded, LIAC 6-12 MeV Sordina mobile linear accelerator. Typically, a single dose of 10-21 Gy was given for maximally resected tumors. The statistical analyses were carried out using SPSS (version 21).

Results: Twenty-six patients were treated with IORT alone or combined with EBRT. Different tumors were treated, including colorectal adenocarcinoma (10 cases, $38.4 \%$ ), Soft Tissue Sarcomas (STS, 11 cases, $42.3 \%$ ), head and neck cancers (3 cases, $11.5 \%$ ), one cervix malignancy case and one paravertebral fibromatosis case. Mean \pm SD overall survival was $15 \pm 14.89(0-38)$ and $34.3 \pm 15.72$ (14-53) months for colorectal cancer and STS, respectively.

Conclusion: IORT is mostly useful for pelvic and abdominal malignancies where normal bowel limits the dose that can be delivered with EBRT. However, the dose delivered in a single fraction with IORT is rarely sufficient for tumor control; therefore, IORT is usually preceded or followed by additional EBRT which should be further evaluated preferably in prospective randomized trials.

Keywords: Intraoperative radiation therapy, External beam radiation therapy, Cancer

Conflicts of Interest: None declared

Funding: None

*This work has been published under CC BY-NC-SA 1.0 license.

Copyright $\odot$ Iran University of Medical Sciences

Cite this article as: Akrami M, Nasrollahi H, Vahabi M, Hamedi SH, Tahmasebi S, Karbasi S, Pashnesaz M, Zangouri V, Karami MY, Mosallaei A, Talei A. Intraoperative radiation therapy in non-breast cancer patients: A report of 26 cases from Shiraz, south of Iran. Med J Islam Repub Iran. 2020 (20 May);34:50. https://doi.org/10.47176/mjiri.34.50

\section{Introduction}

Intraoperative radiation therapy (IORT) is defined as irradiation of the tumor bed or tumor residue during surgery, and can be done by radiotherapy machine or radioactive sources (1). IORT is used as a component of multimodality treatment associated with other local or systemic treatments such as surgery, EBRT and chemotherapy (2).

The best advantage of IORT is sparing the sensitive or-

Corresponding author: Dr Hamid Nasrollahi, nasrolahih@sums.ac.ir

1. Breast Diseases Research Center, Department of Surgical Oncology, Shiraz University of Medical Sciences, Shiraz, Iran

2. Radiation Oncology Department, Shiraz University of Medical Sciences, Shiraz, Iran gans from irradiation and its side effects. Some structures that were or will be irradiated up to the maximum tolerance dose can be spared from toxic doses (1). During IORT, a single large dose of radiation was delivered to the target during surgery in order to decrease the side effects of radiation (3). IORT is used in a wide range of tumors such as breast cancer, sarcoma and gastrointestinal, genitourinary or gynecologic tumors (2).

$\uparrow$ What is "already known" in this topic:

There is no study about IORT effects on non-breast cancer Iranian patients in the medical literature.

$\rightarrow$ What this article adds:

Our results showed the effect of IORT, which is used for primary and local recurrence control of non-breast cancer patients for the first time in Iran. 
Since the mid- $20^{\text {th }}$ century, oncologists and surgeons had tried to use radiotherapy (RT) while performing surgery. The act of RT during surgery can be done by electron, low energy photon or radioactive sources. Of course, protection, transfer of radioactive source, and the chance of infection are considerable. Newer smaller RT devices are made and are easily used in the operation room. Rigid applicators and advanced machines are nowadays available, being able to complete RT procedures in minutes. (4) The aim of the present retrospective study was to report the non-breast cancer patients' IORT outcomes in Iran.

\section{Methods}

As the first report of IORT in non-breast cases in Iran, this is a retrospective study of medical records of patients who had undergone IORT for non-breast malignancies in a 40 months' period in Faghihi hospital, a teaching hospital of Shiraz University of Medical Sciences. IORT was done for both breast and non-breast malignancies in this center from April 2014. By now, more than 400 cases of IORT have been done. Most of the cases are breast cancer, and IORT was done as radical or boost dose. 26 cases who had received IORT had non-breast malignancies.

All cases were selected after they referred to the Shiraz oncology multidisciplinary team (MDT). During the procedure, the radiation oncologist and physicist were present in the operating room to help the surgical oncologist.

All patients had chest, abdomen and pelvic CT scans (or MRI) to rule out lung, liver and other distant metastasis. None of them had metastasis; the only exception was a case of metastatic Ewing sarcoma who had lung metastasis and had a complete response to chemotherapy. All tumors were surgically resected with maximum safe margins (R0 or R1) except for a case with paravertebral aggressive fibromatosis, which has gross residue after operation due to close proximity to the spine and nerve root.

During the surgery for retroperitoneal sarcoma, abdominal cavity was carefully examined to exclude the presence of peritoneal or liver metastases. Patients with intra-abdominal dissemination or distant metastases did not receive IORT.

The IORT treatment volume was determined jointly by the radiation oncologist, physicist and surgical oncologist to cover the regions at the highest risk, including possible tumor residue or tumor bed. IORT was applied by selfshielded, LIAC 6-12 MeV Sordina mobile linear accelerator. Applicators (circular, ellipsoid, or rectangular) in various sizes were used to direct the IORT beam. Typically, a single dose of 10 Gy was given for completely resected tumors; 12.5-15 Gy was delivered for microscopic disease, and 15-20 Gy for macroscopic residual disease. Those patients who were going to receive IORT as the sole treatment received 21 Gy.

The statistical analyses were carried out using Statistical Package for the Social Sciences (version 21; IBM SPSS Inc. Chicago, IL, USA). All data were collected retrospectively; descriptive statistics were generated for all measures, including means, median, ranges, and standard deviations for continuous measures and frequencies and proportions for categorical data.

\section{Results}

Twenty-six patients were treated with IORT. Different tumors were treated, including 10 colorectal adenocarcinoma patients $(38.4 \%), 11$ sarcomas cases $(42.3 \%), 3$ head and neck cancer cases $(11.5 \%), 1$ patient with cervix malignancy $(3.8 \% \%)$, and 1 case of paravertebral fibromatosis $(3.8 \%)$.

\section{Colonic and rectal Cancer}

We treated 10 patients, including 6 men and 4 women with rectal cancer. Six patients had a primary disease and four had recurrent rectal cancer. Mean overall survival was $15(0-38)$ months. No major morbidity happened. Two patients passed away early after the operation due to sudden cardiopulmonary arrest with unknown cause. Five patients received EBRT after operation. At the time of this report, five patients have died and five patients were alive; of them, three patients were disease-free and two were alive with distant metastasis (Table 1).

\section{Soft Tissue Sarcoma}

There were 11 patients with sarcoma that received IORT. They were 6 males and 5 females with a mean age of 46 years. Pathologic types were Liposarcoma (6 cases), malignant fibrous histiocytoma (MFH) (2 cases), Ewing's Sarcoma, Fibrosarcoma, and Undifferentiated sarcoma. There was not any major perioperative complication. Mean overall survival was 34.3(14-53) months. Two patients passed away (Table 2).

\section{Aggressive fibromatosis}

The patient was a 16-year-old boy with unresectable paravertebral aggressive fibromatosis. During the last attempt for resection in our center, IORT (21 Gy) was performed with the purpose of tumor progression and palliation of the pain. He is well for 67 months.

Table 1. Colorectal cancer cases who received IORT

\begin{tabular}{lccccc}
\hline CaSE & Age & Treatment & Treatment & Survival & Patient's Status \\
\hline 1 & 57 & EBRT (44Gy) and IORT (15Gy) & EBRT and IORT & 36 & well Primary \\
2 & 62 & EBRT (44Gy) and IORT (15Gy) & EBRT and IORT & 25 & On chemotherapy \\
3 & 53 & EBRT (44Gy) and IORT (15Gy) & IORT and EBRT & 19 & Passed away due to metastasis \\
4 & 61 & EBRT (44Gy) and IORT (15Gy) & EBRT and IORT & 38 & Well Primary \\
5 & 44 & EBRT (44Gy) and IORT (15Gy) & EBRT and IORT & 23 & With metastasis \\
6 & 45 & EBRT (44Gy) and IORT (15Gy) & EBRT and IORT & 6 & Passed away due to local recurrence \\
7 & 78 & EBRT (44Gy) and IORT (15Gy) & EBRT and IORT & 43 & Recurrence \\
8 & 38 & EBRT (44Gy) and IORT (15Gy) & EBRT and IORT & 3 & Passed away due to metastasis \\
9 & 86 & IORT (15 Gy) & IORT & 0 & Recurrence \\
10 & 74 & IORT (15 Gy) & IORT & 0 & Passed away away \\
\hline
\end{tabular}


M. Akrami, et al.

\begin{tabular}{|c|c|c|c|c|c|c|c|c|c|c|}
\hline Case & Age & Pathology & Location & Treatment & Treatment & Survival & $\begin{array}{l}\text { Tumor } \\
\text { Status }\end{array}$ & $\begin{array}{c}\text { Patient's } \\
\text { Status }\end{array}$ & & $\begin{array}{c}\text { Patient's } \\
\text { Status }\end{array}$ \\
\hline 1 & 32 & $\mathrm{MFH}$ & Retroperuneaum & $\begin{array}{c}\text { EBRT (44Gy) } \\
\text { and IORT } \\
(15 \mathrm{~Gy})\end{array}$ & $\begin{array}{l}\text { EBRT and } \\
\text { IORT }\end{array}$ & 53 & Primary & Well & Primary & $\overline{\text { Well }}$ \\
\hline 2 & 56 & Liposarcoma & Retroperuneaum & $\begin{array}{c}\text { EBRT (44Gy) } \\
\text { and IORT } \\
(15 \mathrm{~Gy})\end{array}$ & $\begin{array}{l}\text { EBRT and } \\
\text { IORT }\end{array}$ & 38 & Primary & Well & Primary & Well \\
\hline 3 & 58 & Liposarcoma & Retroperuneaum & $\begin{array}{c}\text { EBRT (44Gy) } \\
\text { and IORT } \\
(15 \mathrm{~Gy})\end{array}$ & $\begin{array}{l}\text { EBRT and } \\
\text { IORT }\end{array}$ & 39 & Recurrence & $\begin{array}{l}\text { Alive on } \\
\text { Chemo- } \\
\text { therapy }\end{array}$ & Recurrence & $\begin{array}{l}\text { Alive on } \\
\text { chemother- } \\
\text { apy }\end{array}$ \\
\hline 4 & 21 & Fibrosarcoma & Thigh & IORT (21Gy) & IORT & 19 & Primary & $\begin{array}{l}\text { Meta- } \\
\text { static }\end{array}$ & Primary & Metastatic \\
\hline 5 & 61 & $\mathrm{MFH}$ & Thigh & IORT (21Gy) & IORT & 21 & Primary & $\begin{array}{l}\text { Meta- } \\
\text { static }\end{array}$ & Primary & Metastatic \\
\hline 6 & 32 & Ewing sarcoma & Pelvis & $\begin{array}{c}\text { EBRT (44Gy) } \\
\text { and IORT } \\
(15 \mathrm{~Gy})\end{array}$ & $\begin{array}{l}\text { EBRT and } \\
\text { IORT }\end{array}$ & N/A & Recurrence & $\begin{array}{l}\text { Passed } \\
\text { away }\end{array}$ & Recurrence & $\begin{array}{l}\text { Passed } \\
\text { away }\end{array}$ \\
\hline 7 & 40 & Liposarcoma & Thigh & IORT (21 Gy) & IORT & 51 & Primary & Well & Primary & Well \\
\hline 8 & 35 & Liposarcoma & Thigh & $\begin{array}{c}\text { EBRT (44Gy) } \\
\text { and IORT } \\
(15 \mathrm{~Gy})\end{array}$ & $\begin{array}{l}\text { EBRT and } \\
\text { IORT }\end{array}$ & 14 & Primary & Well & Primary & Well \\
\hline 9 & 73 & $\begin{array}{l}\text { Undifferentiated } \\
\text { sarcoma }\end{array}$ & Supraclavicular & $\begin{array}{c}\text { EBRT (44Gy) } \\
\text { and IORT } \\
(15 \mathrm{~Gy})\end{array}$ & $\begin{array}{l}\text { EBRT and } \\
\text { IORT }\end{array}$ & 22 & Recurrence & $\begin{array}{l}\text { Passed } \\
\text { away }\end{array}$ & Recurrence & $\begin{array}{l}\text { Passed } \\
\text { away }\end{array}$ \\
\hline 10 & 41 & Liposarcoma & Retroperuneaum & IORT (21Gy) & IORT & & Recurrence & N/A & Recurrence & \\
\hline 11 & 58 & Liposarcoma & Retroperuneaum & IORT (21Gy) & IORT & & Recurrence & N/A & Recurrence & \\
\hline
\end{tabular}

\section{Cervical cancer}

The other patient was a 46-year-old woman who had recurrent cervical SCC and received IORT (15 Gy) to tumor bed after R2 resection of the lesion. She developed liver and peritoneal metastasis and died 20 months later.

\section{Head and neck cancer}

There were two women with scalp DFSP who received IORT (21 Gy). A 33-year-old woman with recurrent scalp DFSP and a 31-year-old one with newly diagnosed scalp DFSP both received IORT after wide excision of the lesion. Now both of them are well without recurrence after 52 and 61 months. A male patient had parotid SCC and after resection and EBRT (66 Gy) he developed recurrence. He underwent operation and IORT (15 Gy), but he had recurrence again and died 7 months after IORT.

\section{Discussion}

Colorectal cancers are major cancer-related causes of death. EBRT has an established role in the management of rectal cancer. EBRT is used before the operation or is occasionally used after the operation; it improves disease free survival (DFS) and overall survival (OS) (1). Because of anatomic concern, IORT is used in rectal cancer more than colon cancer (1).

Despite multimodality treatment with surgery, EBRT and chemotherapy, local recurrence is a major problem, especially in locally advanced rectal cancer among which the chance of recurrence is $40 \%$ (1). EBRT is usually used in the treatment of rectal cancer and when recurrence occurs, re-irradiation is no more possible. The main obstacle with re-irradiation is damage to such organs as the bowel loops, nerves and vessels. These organs are radiosensitive and when they are irradiated more than a certain level, side effects, including organ failure may be devastating. In these cases, IORT with precise targeted irradiation is a proper choice for radiotherapy (1).

Holman et al. reported 417 patients with T4 rectal cancer who received standard treatment and IORT. While $16 \%$ of patients developed local recurrence, 5 years DFS and OS were $55 \%$ and $56 \%$. Compared to non-IORT reports from their center, IORT improved the local control in locally advanced patients (5)

Some randomized trials were not promising. In a study by Dubois on 142 patients with locally advanced rectal cancers, no survival improvement was seen with IORT. After 5 years, local control was $91.8 \%$ with IORT versus $92.8 \%$ in the standard treatment group. Other oncologic and surgical outcomes including metastasis and complications were not statistically significant in both groups. Other studies showed no benefit of IORT in rectal cancer (1, $6,7)$. While up to $8 \%$ perioperative mortality has been reported in a review on IORT in colorectal cancer, we had 2 deaths that were related to cardiac problems.

We treated 10 patients with colorectal cancer who received IORT. Only 3 patients were disease-free. We had 4 patients with recurrence among them; 3 of them died, and 1 patient is alive and is receiving chemotherapy. It seems that IORT must be chosen more carefully in these patients. We suggest that IORT should be done in isolated local recurrence in rectal cancer.

Cervical cancer is radiosensitive and also has a dosedependent response rate. Most recurrences of cervical cancer occur in the pelvic cavity and about $60 \%$ of patients have local recurrence at the time of death (8). Barney et al. in a report on 86 patients with recurrent $(n=73$, $85 \%)$ or primary advanced cervix cancer $(n=13,15 \%)$ administered IORT. Local recurrence after 3 years was $23 \%$. In statistical analysis, IORT had no relation to the recurrence (8). We had one case of cervical cancer and she 
passed away 20 months after the treatment with IORT.

Recurrence occurs in $20-30 \%$ of patients with extremity STS. Although the outcome after recurrence is less favorable than primary sarcomas, the treatment protocols are the same as the primary tumors. In those patients with recurrent sarcoma of extremities, surgery and reirradiation, if possible, is the treatment of choice, but reirradiation has severe toxicities (9).

In a study by Carbo et al. on 39 patients with primary (33 patients) or recurrence (6 patients) extremity STS received IORT. 33 patients received IORT and EBRT, and 6 received IORT alone. Local recurrence occurred in $18 \%$ of patients. Those with recurrent sarcoma had a 50\% chance of the second recurrence, while in primary sarcoma, the recurrence rate was $12 \%$. Margin status was a significant factor in recurrence. While $50 \%$ of patients with involved margins had local recurrence, only $7 \%$ of those with negative margins had recurrence. They concluded that local control was excellent, and side effects were acceptable (10). IORT provides an excellent chance to irradiate the tumor bed or residue and spare the sensitive tissues. Tinkle reported 26 patients with recurrence of extremity STS who received IORT during surgery for recurrence. Margins were close or microscopically involved in 19 patients. 10 patients developed local recurrence with a median time of 10 months. Estimated 5 years' local control was $58 \%$. The main acute and late significant side effects were musculoskeletal related (9). We had 4 cases of lower extremity sarcoma who received IORT due to recurrence. Two cases developed metastasis and passed away and 2 other cases are well and alive now.

Although retroperitoneal sarcoma is less common than extremity sarcoma, recurrence is more prevalent. Radiation can improve the disease control and may be administered before or after the operation. Retroperitoneal sarcoma does not have a good prognosis. In studies, 5 year local control is $50 \%$ and 10 year survival is about $5 \%$ ( 11 , 12).

Due to low incidence rate, information on IORT in retroperitoneal sarcoma is not sufficient. In a retrospective study on 908 patients with retroperitoneal sarcoma, 33 patients received IORT alone and 32 patients received IORT and EBRT. Mean OS in patients with EBRT without IORT was 52.6 months. Those who received IORT as a part of treatment had 59.4 months OS and this difference was not statistically significant. Survival in those who received combination of IORT and EBRT was significantly better than IORT alone or EBRT alone (87, 34 and 55 months, respectively) (11). We had 5 patients with retroperitoneal sarcoma. Two cases had lost their F/U. 2 cases are well and alive after 53 and 58 months who received EBRT and IORT. One of them received IORT for the recurrence and after 39 months is alive with metastasis. Regarding the anatomical condition of the retroperitoneal area, further studies are required to establish a standard method for IORT.

Fibromatosis is a nonmalignant disease with no ability for metastasis. RT may be needed in some situations. In a study by Roeder, IORT was used in fibromatosis in 30 patients. Recurrence occurred in 5 cases, among whom 3 cases had recurrence in the IORT field. 5-year disease control was $82 \%$. The authors suggested IORT as an effective modality in aggressive fibromatosis (13). We had a 16-year-old patient with unresectable aggressive fibromatosis whose lesion was close to the spine. In order to avoid RT to the spine and normal tissue, IORT was done for him, and he has been well for 52 months.

About one-third of patients with head and neck cancers may develop local recurrence. Local recurrence in these patients is a catastrophe. In a study carried out by Wild, 61 patients were evaluated. $72 \%$ of them had SCC and other patients had none-SCC. Mean overall survival was 19 months, and median progression-free survival was less than 10 months (14). Nag et al. in another retrospective study, reported their experience with IORT in head and neck cancers. 38 patients who had received EBRT received IORT at recurrence. $66 \%$ of the patients developed recurrence at the site of IORT in a median time of 6 months. In this study, complications were severe and included orocutaneous fistulae, wound dehiscence, carotid occlusion, and fatal tracheovascular fistula which occurred in $16 \%$ of patients. Survival was not satisfactory and 1-, 2 , and 3-year survival rates were $21 \%, 21 \%$, and $8 \%$, respectively $(15,16)$. Our case had recurrent right parotid SCC. He underwent radical resection and IORT and developed locoregional recurrence again and unfortunately died 7 months later.

DFSP is a benign-looking disease and at presentation may be mistaken by lipoma or other similar diseases. DFSP is usually seen in the trunk and scalp is not a common site for DFSP, but treatment may be challenging, especially in the scalp. DFSP may be an aggressive lesion and especially in the scalp, reconstruction is a problem. The main treatment is surgery and RT is reserved for especial conditions. A wide margin of resection is needed and in cases with a margin larger than $4 \mathrm{~cm}$, there is still a $6 \%$ chance for recurrence (17). Radiation is suggested in patients with positive margins or recurrence (18). We had two cases of DFSP of the scalp. They are well after 52 and 61 months without recurrence.

The limitation of our case series study is the lack of enough sample size, so authors could not examine disease etiology and outcome. The present study outcome should be compared with non-IORT cases in the prospective study to assess reliable results.

\section{Conclusion}

IORT is most useful for pelvic and abdominal malignancies where normal bowel limits the dose that can be delivered with EBRT. However, the dose delivered in a single fraction with IORT is rarely sufficient for tumor control; therefore, IORT is usually preceded or followed by additional EBRT, which should be further evaluated preferably in prospective randomized trials.

\section{Acknowledgments}

The authors would like to thank Shiraz University of Medical Sciences, Shiraz, Iran, and the Center for Development of Clinical Research of Nemaazi Hospital for their support. We also thank Dr. Nasrin Shokrpour for editorial 
assistance.

\section{Conflict of Interests}

The authors declare that they have no competing interests.

\section{References}

1. Mirnezami R, Chang GJ, Das P, Chandrakumaran K, Tekkis P, Darzi $\mathrm{A}$, et al. Intraoperative radiotherapy in colorectal cancer: systematic review and meta-analysis of techniques, long-term outcomes, and complications. Surg Oncol. 2013;22(1):22-35.

2. Pilar A, Gupta M, Ghosh Laskar S, Laskar S. Intraoperative radiotherapy: review of techniques and results. Ecancermedicalscience. 2017;11:750.

3. Krengli M, Pisani C, Deantonio L, Surico D, Volpe A, Surico N, et al. Intraoperative radiotherapy in gynaecological and genito-urinary malignancies: focus on endometrial, cervical, renal, bladder and prostate cancers. Radiat Oncol J. 2017;12(1):18.

4. Paunesku T, Woloschak GE. Future Directions of Intraoperative Radiation Therapy: A Brief Review. Front Oncol. 2017;7:300--

5. Holman FA, Haddock MG, Gunderson LL, Kusters M, Nieuwenhuijzen GA, van den Berg HA, et al. Results of intraoperative electron beam radiotherapy containing multimodality treatment for locally unresectable T4 rectal cancer: a pooled analysis of the Mayo Clinic Rochester and Catharina Hospital Eindhoven. J. Gastrointest Oncol. 2016;7(6):903-16.

6. Dubois JB, Bussieres E, Richaud P, Rouanet P, Becouarn Y, Mathoulin-Pelissier S, et al. Intra-operative radiotherapy of rectal cancer: results of the French multi-institutional randomized study. Radiother Oncol. 2011;98(3):298-303.

7. Rangarajan K, Bhome R, Bateman N, Naga A, Simon M, Donovan K, et al. Pelvic exenteration with en bloc resection of the pelvic sidewall and intraoperative electron beam radiotherapy with Mobetron $(\circledR)$ for locally advanced rectal cancer. Tech Coloproctol. 2017;21(6):493-5.

8. Barney BM, Petersen IA, Dowdy SC, Bakkum-Gamez JN, Klein KA, Haddock MG. Intraoperative Electron Beam Radiotherapy (IOERT) in the management of locally advanced or recurrent cervical cancer. Radiat Oncol J. 2013;8:80.

9. Tinkle CL, Weinberg V, Braunstein SE, Wustrack R, Horvai A, Jahan $\mathrm{T}$, et al. Intraoperative Radiotherapy in the Management of Locally Recurrent Extremity Soft Tissue Sarcoma. Sarcoma. 2015;2015:913565.

10. Carbo-Laso E, Sanz-Ruiz P, Calvo-Haro JA, Cuervo-Dehesa M, Perez-Mananes R, Mediavilla-Santos L, et al. Intraoperative radiotherapy for extremity soft-tissue sarcomas: can long-term local control be achieved? Int J Clin Oncol. 2017;22(6):1094-102.

11. Wang LB, McAneny D, Doherty G, Sachs T. Effect of intraoperative radiotherapy in the treatment of retroperitoneal sarcoma. Int $\mathrm{J}$ Clin Oncol. 2017;22(3):563-8.

12. Roeder F, Krempien R. Intraoperative radiation therapy (IORT) in soft-tissue sarcoma. Radiat Oncol. 2017;12(1):20-.

13. Roeder F, Timke C, Oertel S, Hensley FW, Bischof M, Muenter $\mathrm{MW}$, et al. Intraoperative electron radiotherapy for the management of aggressive fibromatosis. Int $\mathrm{J}$ Radiat Oncol Biol Phys. 2010;76(4):1154-60.

14. Wald P, Grecula J, Walston S, Wei L, Bhatt A, Martin D, et al. Intraoperative electron beam radiotherapy for locoregionally persistent or recurrent head and neck cancer. Head Neck-J Sci Spec. 2019;41(7):2148-53.

15. Nag S, Schuller DE, Martinez-Monge R, Rodriguez-Villalba S, Grecula J, Bauer C. Intraoperative electron beam radiotherapy for previously irradiated advanced head and neck malignancies. Int $\mathrm{J}$ Radiat Oncol Biol Phys. 1998;42(5):1085-9.

16. Kyrgias G, Hajiioannou J, Tolia M, Kouloulias V, Lachanas V, Skoulakis $\mathrm{C}$, et al. Intraoperative radiation therapy (IORT) in head and neck cancer: A systematic review. Medicine (Baltimore). 2016;95(50):e5035-e

17. Bhatnagar A, Srivastava A, Sahu RN. Management of Recurrent Dermatofibro Sarcoma Protuberance of Scalp-a Reconstructive Challenge. Indian J Surg Oncol. 2013;4(1):15-8.

18. Uysal B, Sager O, Gamsiz H, Cicek A, Demiral S, Dincoglan F, et al. Evaluation of the role of radiotherapy in the management of dermatofibrosarcoma protuberans. J BUON. 2013;18(1):268-73. 